\title{
Ethnicité et pouvoir au Nord-Cameroun ${ }^{1}$
}

\author{
Par Ibrahim Mouiche
}

«Le Cameroun se révèle une terre de la multiplicité et de la division socio-historique, le lieu des rendez-vous d'une variété insoupçonnable des forces centrifuges et antagonistes crampant face à face en une sorte de veillée d'armes permanente où l'évidence des particularismes est par trop évidente.» ${ }^{2}$

Centre de gravité du continent africain, le Cameroun ainsi que le décrit si bien Jean Imbert $^{3}$, peut être considéré comme une Afrique en réduction et son étude est particulièrement bénéfique pour qui veut s'initier aux problèmes africains. Car, au point de jonction des régions géographiques occidentale, septentrionale et centrale, le territoire camerounais est le carrefour où se rencontrent trois importantes régions culturelles: la Côte du Guinée avec ses peuplades négritiques, le Soudan Occidental avec les Peuls et les peuplades arabes, le Congo avec les peuples de langue bantou. L'extrême complexité de la configuration ethnique est à l'image de celle de l'Afrique. C'est pour cette raison que certains auteurs utilisent l'expression «Habit d'Arlequin» pour caractériser une telle diversité.

En fait, le Cameroun rappelle étrangement l'ex-Yougoslavie dont la situation peu enviable peut se résumer en quelques chiffres ainsi que le stigmatise M. Jean-Pierre Fogui ${ }^{4}$ : deux alphabets (latin et cyrillique), trois grandes religions (orthodoxe, catholique et musulmane), cinq nationalités (Serbe, Croate, Slovène, Macédonienne et Monténégrine), six Républiques (Serbie, Croatie, Macédoine, Monténégro, Bosnie-Herzégovine), sept voisins (Italie, Autriche, Hongrie, Roumanie, Bulgarie, Grèce et Albanie).

Le «Nord» du Cameroun est une zone de prairie tropicale au climat soudano-sahélien qui ne présente aucune unité géographique. Il comprend depuis 1983 trois provinces: l'Adamaoua avec une population évaluée en 1991 'a 471.000 habitants, le Nord avec 672.000 habitants et l'ExtrêmeNord avec 1.896.000 habitants (voir l'essentiel sur le Vie plan quinquennal de Développement Economique, Social et Culturel, 1986-1991, Yaoundé, Imprimerie Nationale, p. 32). Cette zone comprise entre les parallèles $6^{\circ} \mathrm{N}$. et $12^{\circ} \mathrm{N}$, représente $157.000 \mathrm{~km}^{2}$, soit plus du tiers de la superficie de la République du Cameroun et 30,3\% de la population.

2 Paul Biya, Pour le Libéralisme Communautaire, Paris, 1978, p. 33

3

Imbert, J., Le Cameroun, Paris, 1973, p. 3, lere Edition, Collection Que sais-je?

4 Fogui, J.-P., L'intégration politique au Cameroun. Une analyse centre-périphérie, Paris, 1990, pp. 41-42. 
La carte d'identité du Cameroun est tout aussi éloquente: deux langues officielles (français et anglais), deux Etats fédérés jusqu'en 1992 (Cameroun occidental anglophone et le Cameroun oriental francophone), trois colonisations (allemande, française et anglaise), plus de deux cents ethnies et toutes les races africaines.

Quant à l'idée ethnique, elle jouit d'un large consensus dans le paysage politique africain. Ce qui a fait dire à Jean François Bayart que «l'espace politique contemporain de facture étatique s'est moins construit par l'érosion des identifications particulières héritées de l'Afrique ancienne que par la fabrication de nouvelles identités particulières; spécialement celles de l'ethnicité. L'Islam et le Christianisme ont été des véhicules éminents de cette ethnogenèse»" 5 .

D'ailleurs, il ne manque d'examples pour démontrer que les institutions politiques, souvent l'ensemble des systèmes politiques sont façonnés par des divisions ethniques. Ainsi le fond fédéral de la République du Nigéria résulte avant tout d'une division reconnue entre les Yorouba, les Ibo et les Haoussa-Foulani, chaque groupe occupant une zone géographique assez bien délimitée, fait de la plus haute importance, exerçant une influence décisive sur les institutions de leurs zones respectives. ${ }^{6}$

Donc, entre le pouvoir et l'ethnicité, il existe une indissoluble connexité, encore que ces termes soient d'usage courant non seulement dans les discours des sciences sociales mais également dans la langue de tous les jours. Cet usage mérite cependant de précision. Nous allons donc nous efforcer d'apporter un certain nombre de clarifications d'ordre conceptuel et nous interroger sur les relations dialectiques entre les deux concepts.

Si, par ethnie nous entendons un nombre considérable de personnes qui, du fait de leurs caractéristiques culturelles communes et des relations étendues entre elles, se considèrent et sont considérées comme une entité culturelle ${ }^{7}$, l'ethnicité serait donc une affaire de culture. Or le fait d'appartenir à une culture différente n'implique pas une supériorité ou une infériorité; il n'est pourtant pas rare que certaines communautés se considèrent comme culturellement supérieures à d'autres. C'est le problème qui oppose au Nord-Cameroun et depuis longtemps les Peul aux non-Musulmans communément appelés «Kirdi» ${ }^{8}$. Certains auteurs

5

Bayart, J.F., Religion et modernité politique: Dieu pour tous et chacun pour soi, Paris, 1993, p. 14.

6 Le Vine, V.T., Le Cameroun, Paris, 1970, tome 1, p. 26.

7 Su Neba, A., Géographie moderne de la République du Cameroun, Camden (New Jersey), 1987, p. 45 .

8 Kirdi: terme de langue arabe Choa du Ouadaï et du Baguirmi signifiant «infidèle», c'est-à-dire «non musulman»- Ce vocable est usité par les Peul (Kaado ou Habé) pour désigner les ethnies païennes du Nord-Cameroun. 
anglo-saxons ont donc proposé le terme d'«ethnicisme» (ethnicism) par référence au racisme pour désigner les discriminations fondées sur l'ethnicité. ${ }^{9}$ Cette dernière est quant à elle, considérée de plus en plus comme un phénomène positif, une marque d'identité, une quête de racines. Par conséquent, ethnicité et ethnicisme désignent les deux faces, l'une positive, l'autre négative de l'identité ethnique. ${ }^{10}$ Et l'ethnicité semble ainsi légitime puisque les gens peuvent revendique ouvertement une identité ethnique quelconque, s'en montrer fiers et en bien des cas se mobiliser activement en faveur de la suppression des inégalités dont ils estiment être victimes. ${ }^{11}$ Ce que font les Kirdi (depuis l'ouverture démocratique) au Nord-Cameroun.

Marquée ainsi par l'ethnocentrisme, une conscience et une identité communes ainsi que par l'exclusivisme, l'ethnicité à l'instar de tout phénomène social, est sujette à des mutations. Elle peut changer de forme, de place et de rôle dans la vie d'une société. Son contenu peut s'enrichir de nouveaux éléments, ses liens avec d'autres phénomènes sociaux peuvent se modifier et ce faisant, être su jets à de nouvelles questions.

Par ailleurs, l'ethnicité à l'état pur n'existe pas, elle est toujours étroitement associée à des considérations d'ordre politique, juridique, religieux et social qui constituent parallèlement ses composantes essentielles. ${ }^{12}$ Dans le cas particulier du Nord-Cameroun, l'islamisation des populations kirdi a souvent été un prétexte d'ascension sociale et d'intégration dans le «bloc de pouvoir» ${ }^{13}$ musulman, peul.

Donc, l'ethnie en tant qu'entité homogène et correspondant à un territoire délimité n'existe pas. Comme toutes les identifications culturelles, la conscience ethnique est contextuelle et se définit par rapport à l'autre (et par le regard de l'autre). En outre, elle n'est pas exclusive d'identifications complémentaires ou concurrentes, telles que les identifications à des lignes de différentiations ancestrales ou nées de la division du travail ou encore à des ensembles culturels supra-ethniques d'ordre religieux ou national. Elle véhicule ainsi des représentations autres que «ethniques» que interdisent de réduire les affrontements de ce type à de simples conflits désincarnés d'identifications. Y sont également en jeu les intérêts politi-

9 Bacal, A., Ethnicity in the social sciences, Coventry, University of Warwick, 1991.

10

11

12

13 
ques, religieux, économiques. En omettant de le préciser, l'interprétation classique en terme de «tribalisme» les rend aussi inintelligibles. ${ }^{14}$

En définitive, la dialectique des identités ethniques et les enjeux politiques «nationaux» de l'Etat (les confrontations et concurrences forcées ou obligatoires) est d'abord une dialectique de la stratégie et de l'alliance, le contrat social ne s'exprimant pas encore sous la forme d'une identité formelle de la représentation politique (donc de la représentativité sociologique). C'est cette discordance qui superpose les causes du conflit et donne à la scène africaine cet aspect brouillon ${ }^{15}$ puisque la communauté ethnique est un chenal par lequel est revendiquée la redistribution ainsi qu'un instrument d'accumulation comme au Nord-Cameroun où les conflits ethniques sont nourris par les clivages socio-économiques et religieux en vue de l'acquisition du pouvoir et donc de la richesse.

Si l'ethnicité est ainsi un instrument du pouvoir, ce dernier pour être opérationnel dans notre contexte ne doit pas être pris comme le simple produit (à la limite pathologique), et la preuve de la prégnance d'une structure d'autorité, d'un mode de domination sociale mais plutôt comme l'entend Michel Crozier, c'est-à-dire «le résultat toujours contingent de la mobilisation par les acteurs des sources d'incertitudes permanentes qu'ils contrôlent dans une structure de jeu donnée pour leurs relations et tractations avec les autres participants à ce jeu. C'est donc un embryon d'action impliquant marchandage et intégration, une relation qui, en tant que médiation spécifique et autonome des objectifs divergents des acteurs est toujours liée à une structure de jeu» 16 .

Domineront alors ceux des acteurs qui seront capables d'affirmer et d'imposer leur maîtrise des sources d'incertitudes les plus cruciales. Le pouvoir réside donc dans la marge de liberté dont dispose chacun des partenaires engagés dans une relation de pouvoir, c'est-àdire dans sa possibilité plus ou moins grande de refuser ce que l'autre lui demande. Et la force, la richesse, le prestige, l'autorité bref les ressources que possèdent les uns et les autres n'interviennent que dans la mesure, où ils leur fournissent une liberté d'action plus grande. ${ }^{17}$ C'est donc le prophète chargé de formuler l'ordre nouveau, d'imaginer de nouvelles cohérences destinées à remplacer les anciennes. C'est aussi l'instituteur chargé de faire comprendre, de faire accepter l'ordre nouveau. Enfin le pouvoir doit être le gendarme

Bayart, J.F., Les sociétés africaines face à l'Etat, in: Revue française d'études constitutionnelles et politiques, ${ }^{\circ} 5,1983$, p. 27.

15 Voir J. Copans, La longue marche de la modernité africaine. Savoir, intellectuels, démocratie, Paris, 1990, p. 186.

16

Crozier, M., L'acteur et le système, Paris, 1974, p. 27.

17

Ibid., pp. 59-60. 
chargé de neutraliser les récalcitrants ou de forcer les égarés, s'il en trouve, à se conformer à l'ordre nouveau. ${ }^{18}$

Au Nord-Cameroun, le pouvoir a pendant longtemps été utilisé par les musulmans sous la houlette des Peul pour opprimer les populations païennes (Kirdi) qui aujourd'hui oeuvrent pour la déconstruction de cet ordre déphasé dans le train du Président Biya, un homme du «Sud».

En effet, sous la présidence de M. Ahidjo (un Peul) s'est propagée l'image du «Nord» comme une société homogène, unie sous le commandement des musulmans parmi lesquels les Peul constituent l'ethnie dominante ${ }^{19}$ au contraire du «Sud» chrétien, multiethnique et animiste; véritable fantasme qui, pendant longtemps a dévoyé des esprits peu curieux (tout Nordiste étant considéré comme musulman ou mieux un Peul) puisque le Nord dont il est originaire constituait une base-arrière politique solide pour lui face au «Sud». Politique suicidaire pour les populations non-islamisées mais qui trouve leurs racines dans l'histoire des peuplements du Nord-Cameroun depuis le XIXe siècle renforcées sous la colonisation allemande et française.

Or, en prenant en considération le point de vue du citoyen ordinaire du Nord (l'homme de la rue ou l'homme de la brousse) tel qu'on peut le saisir par des moyens classiques (observation, enquêtes etc...), dans les manifestations les plus explicitement politiques (à l'occasion des élections par exemple) mais aussi tel qu'on peut le deviner dans des contextes où, de plus en plus souvent, il lui est difficile de 'exprimer ouvertement, par l'analyse culturelle et symbolique, on ne peut que s'étonner de la diversité ethnico-religieuse de cette région du pays dont se sont toujours servis les pouvoirs publics au gré des convulsions politiques.

Quand le Président Biya arrive au pouvoir en 1982, il est de plus en plus secoué par le joug musulman pro-Ahidjo. Il s'active à démanteler l'hégémonie peul-musulmane en divisant le Nord en trois provinces, s'appuie sur les Kirdi concentrés en majorité dans la province de l'Extrême-Nord, la plus peuplée du pays non sans les avoir émancipés. Et depuis la restauration du multipartisme, les Kirdi soutiennent le parti du R.D.P.C. du Président Biya, repoussent l'U.N.D.P., le parti néo-ahidjoïste du Peul Bouba Bello Maïgari. Ce qui les propulse au devant de la scène politique nationale à commencer par l'échiquier régional.

Gonidec, P.F., Traditionalisme et modernisme en matière d'institutions publiques africaines, in: Revue juridique et politique, indépendance et coopération, $\mathrm{n}^{\circ} 1$, janv.-mars, 1966, p. 78. n41, Paris, 1991; voir également Dakolé Daïssala, Libre derrière les barreaux, Paris, 1993. 
Donc, les différentes ethnies du Nord-Cameroun ne vivent pas dans l'autarcie. Elles sont traversées par des courants centripètes et centrifuges, par un mouvement de répulsions et d'interdépendance. Notre interrogation portera alors sur la diversité ethnique du NordCameroun, l'hégémonie musulmane et les regroupements politiques de cette région le tout mû par la logique d'accumulation.

\section{Diversité ethnique, brassage des populations et hégémonie musulmane au Nord-Cameroun}

Le Nord-Cameroun est parcouru des clivages sociaux dont il est simpliste de réduire à une contradiction entre une minorité dominante peul et une majorité dominée kirdi. ${ }^{20}$ Certes, ces distinctions existent. Et elles jouent un rôle indéniable dans la constitution des identités culturelles et politiques. Mais dans les faits, ces identités sont multiples tout comme les loyautés qu'elles appellent et les formes de l'inégalité dont elles s'accommodent. Il est vrai qu'aucun Peul ne se dirait non musulman, mais l'adhésion de certains d'entre eux (les pasteurs Bororo par exemple) à la foi du prophète reste superficielle. Quant aux Kirdi ${ }^{21}$ ils représentent une mosaique humaine socialement hétérogène dont l'insertion au système régional d'inégalités varie d'un groupe à l'autre. L'équation du Nord se laisse plutôt ramener à l'hégémonie d'un bloc au pouvoir, cimenté culturellement par l'«islam way of life», mais ethniquement hétéroclite puisque l'on y retrouve les grands notables foulbé, les commerçants haoussa, les Kotoko, les Bornouan et les «élites» converties Kirdi. ${ }^{22}$

Par ailleurs, même s'il est mû par des intérêts communs, le «bloc» musulman est loin de présenter l'homogénéité qu'on lui prête. La compétition entre les lamidats ${ }^{23}$ et les milieux d'affaires de Ngaoundéré, de Garoua et de Maroua précède la colonisation. Rey-Bouba a toujours marqué son autonomie contestant de fait la prééminence des grands centres urbains. De même, il est des musulmans comme les Arabes Choa de l'Extrême-Nord qui ont été marginalisés et n'occupent pas une position socio-politique privilégiée. ${ }^{24} \mathrm{Et}$ même si

L'appellation ancienne des populations païennes (Kirdi) n'est plus exacte puisque beaucoup se sont convertis à l'islam et au christianisme.

Contrairement à l'idée communément admise, les Kirdi constituent l'ossature numérique de la région puisqu'ils représentent les 70 à $73 \%$ de la population au contraire des musulmans confinés dans les proportions de 27 à $30 \%$. Voir sur ce point l'ouvrage collectif de l'ORSTOM, Le NordCameroun, des homme, une région, Paris, 1984.

Voir Bayart, J.F., L'Etat en Afrique, La politique du ventre, Paris, 1989, p. 17.

Voir Mbembe, A., Crise de légitimité, restauration autoritaire et déliquescence de l'Etat, in: Peter Geschiere / Piet Konings, Itinéraires d'accumulation au Cameroun, Pathways to accumulation in Cameroon, Paris, 1993, p. 351. 
l'on peut considérer que le fait d'adhérer à l'islam crée entre les islamisés une communauté de pensées, d'attitudes, de «civilisations» et relègue au second plan les particularismes ethniques, le seul fait de s'opposer à l'islam et de résister à toute forme d'assimilation ne suffit pas à créer la même uniformité entre les groupes kirdi qui souvent s'ignorent, parfois s'opposent et dans tous les cas s'efforcent de préserver leur originalité. ${ }^{25}$

\section{A. Les différents groupes ethniques du Nord-Cameroun et leurs brassages}

Compte tenu de la complexité du peuplement et l'imbrication des groupes, il n'est possible de s'en tenir à une population isolée. Les ethnies qui occupent rarement leur territoire d'origine sont difficiles à définir et si l'on peut accepter l'idée qu'elles constituent un groupe d'individus qui partagent momentanément une communauté linguistique, culturelle et sociale plus élevée que celle qu'ils partagent avec ses voisins, les groupes bâtards sont nombreux. Ce d'autant plus que l'histoire du peuplement du Nord suggère d'innombrables emprunts et assimilations, les populations ayant de profondes affinités dans le domaine de l'organisation sociale et religieuse. ${ }^{26}$

Pas plus qu'il n'est possible de considérer les sociétés comme des monolithes, il n'est possible de s'en tenir à des limites géographiques rigides et il est bien certain qu'une tentative de reconstruction historique dans cette partie de l'Afrique doit prendre en considération les régions voisines. Ce qui implique une étroite collaboration entre le Cameroun, le Tchad, la République Centre Africaine et le Nigéria. ${ }^{27}$

Les populations du Nord sont rangées globalement et abusivement sous deux étiquettes: les musulmans des plaines et les Kirdi des montagnes (Monts Mandara et Atlantika). Néanmoins on retrouve une forte colonie Kirdi dans les plaines (notamment dans les zones situées entre les Monts Mandara et la rivière Logone et de mesas au Sud de la Bénoué): les Moundang, les Guiziga, les Toupouri et les Mousgoy sans oublier ceux de l'Adamaoua (Mboum, Laka, Gbaya, Dourou etc...).

Les ethnies musulmanes sont: les Mandara, les Foulbé, les bornouan, les Kotoko, les Haoussa et les Arabes Choa. Elles sont localisées à l'Extrême-Nord dans les lamidats de Dumru, Guidiguis et dans les plaines du Diamaré et de Mora. Dans le Nord, les Foulbé se retrouvent dans les lamidats de Golombé et Mayo-Ulo tandis que les lamidats de Lam, Guider, Bidzar concernent surtout les animistes Guidar, Fali, Moundang, Gondé, etc... A

25

26

27

Ibid.

Voir Pontié, Guy, Les Guiziga du Cameroun Septentrional, Paris, ORSTOM, 1973, p. 7.

Voir de Carne, I., Contribution à l'histoire du Mayo Danaye (Massa, Toupouri, Moussey et Mousgoum), in: $C$. Tardits, Contribution de la recherche ethnologique à l'histoire des civilisations du Cameroun, Paris, 1981, tome 1, p. 171. 
Dourbey, le milieu est aussi dominé par les Kirdi, les musulmans n'apparaissant que dans des voyers spécifiques. ${ }^{28}$

Pour mieux démêler l'écheveau des groupes ethniques du Nord-Cameroun, il importe de dépasser la division musulmans/Kirdi tant les ethnies sont nombreuses qu'une telle classification paraît réductrice. Aussi distinguerions-nous à la suite de Aaron Su Neba ${ }^{29}$ les Soudanais, les Foulbé (groupes principaux) ${ }^{30}$ et le groupe minoritaire Arabe Choa.

\section{Les Soudanais}

Ce sont les premiers habitants du Nord-Cameroun. Parmi eux l'on distingue les PaléoSoudanais et des Néo-Soudanais.

\section{a) Les Paléo-Soudanais}

Ils sont les tout premiers à occuper le Nord. Chassés après un certain temps par les NéoSoudanais et les Foulbé, ils se réfugient dans les hautes terres d'où ils pourront facilement se défendre. Aujourd'hui, ils sont présents dans les terres s'étirant des Monts Mandara et Atlantika jusqu'à la limite Sud du bassin de la Bénoué. ${ }^{31}$

Les Monts Mandara septentrionaux, les plus densément peuples forment une véritable mosaïque ethnique où quelques groupes, les Mafa et les Mofu ${ }^{32}$, dominent numériquement un ensemble de groupes aux dimensions restreintes: les Podoko, les Mora, les Vamé, les Mbrémé, les Mouyeng, les Ouldémé, les Mada, Les Mokyo, les Zouglo, les Guemzok, les Mbokou etc..., tous subdivisés en multiples clans.

Ce fractionnement ethnique extrême témoignage de l'importance et du nombre de chocs politiques et naturels qui ont atteint le coeur des montagnes jusqu'aux Monts Mandara

Akam, M., Le lamidalisme dans la problématique du développement rural au Nord-Cameroun, thèse sociologie, Bordeaux II, 1984, p. 6.

Neba, op. cit. (note 7), p. 57.

Surce point voir Froelich, J.C., Le commandement et l'organisation sociale chez les Fali du NordCameroun, I.F.A.N., Etudes camerounaises, oct.-déc. 1956, p. 20.

Beauvilain, A., Nord-Cameroun: Crises et Développement, thèse de doctorat ès lettres et sciences humaines, Université de Rouen, 1989, tome 1, p. 22.

Ce terme arbitraire rassemble deux groupes ethniques différents: l'un au Nord sur onze massifs et l'autre au Sud sur seize massifs. Cette division est perçue de l'intérieur par de nombreux traits culturels (habitats, costumes, poteries etc.) et par de différences au niveau des institutions. Cf. Vincent, J.Y., Eléments d'histoire des Mofu, Montagnards du Cameroun, in: Tardits, op. cit. (note 26), p. 273. 
méridionaux puisque les Kapsiki ${ }^{33}$ et les Bana s'y reconnaissent un métissage avec les Mofou $^{34}$. De même les Daba ${ }^{35}$, les Guiziga, les Kapsiki, les Mafa et les Mofou auraient en commun une étape migratoire à Goudour, massif situé sur la bordure orientale des Monts Mandara. Toutefois il faudrait tenir compte de la fonction religieuse jouée par Goudour pour expliquer tous ces rattachements réels ou supposés à ce lieu. Centre de Pélerinage, Goudour était en quelque sorte «La Mecque» des animistes. ${ }^{36}$

Dans les Monts Mandara méridionaux et leurs piedmonts, le morcellement ethnique paraît moins fort et les densités plus faibles derrière quelques masques généraux comme celui du Fali, terme appliqué à des agglomérats des populations aux origines fort diverses et que la conquête peul a moulés dans une histoire commune dans le département de la Bénoué. De

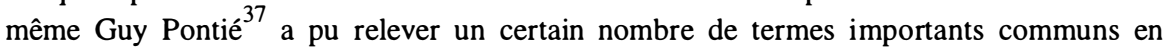
langues Guiziga, Mofou, Matakam, preuve sinon d'apparentement du moins des contacts antérieurs étroits entre ces ethnies. Les Sao restés en place et métissés avec leurs envahisseurs massa donnèrent naissance aux Kotoko. Les Sao ayant disparu en tant que nation, il semble que certains groupes massa aient également atteint dans leur marche vers l'ouest la région de Dulo (au Nord de Mora) alors habitées par les païens Soudanais. De la fusion des premiers occupants et des Massa seraient nés les actuels Mandara. On recoupe ici la légende donnant une origine commune aux Mandara et aux païens Podoko ${ }^{38}$.

\section{b) Les Néo-Soudanais}

Ils occupent les plaines septentrionales et particulièrement le bassin du Logone. Dans ce groupe on distingue les Massa, les Mousgoum, les Mousouk, les Moussey, les Toupouri

33

Le groupement kapsiki du Nord-Cameroun et le groupement higi du Nigéria ont été considérés comme une même ethnie. Il est difficile de parler d'une ethnie Kapsiki proprement dite. Les villages sont autonomes, ont peu de rapports entre eux et se distinguent par de différences marquées. Voir van Beek, W.E.A., Les Kapsiki, in: Tardits, op. cit. (note 26), p. 113.

34

35

36

37

38

Guy Pontié, op. cit. (note 25), pp. 7-9.

Lembezat, Kirdi, les populations païennes du Nord-Cameroun, mémoire de l'IFAN, série population, $\mathrm{n}^{\circ} 3,1950$, p. 19 
sans oublier les Dourou, les Mboum et les Laka (apparentés aux Mboum) de l'Adamaoua, peuples cultivateurs en interaction permanente.

Les affinités ethniques nous permettent de rattacher les Moundang aux Toupouri, leurs voisins immédiats et aux Mboum qui auraient, selon certaines traditions été les premiers occupants de la vallée du Mayo Kebi. Sur le plan de la langue parlée, les assimilations sont fréquentes. La franche Nord du canton de Yagoua est occupée par des groupes d'origine Mousouk qui ont adopté la langue et la culture matérielle des Massa et l'on est bien embarrassé pour classer les Bahigana avec les Massa ou avec les Mousgoum. Selon Mouchet ${ }^{40}$ les groupes résiduels toupouri, les Végué sont discernables des Massa Walia au sein desquels ils vivent. Des villages d'origine mousouk ayant adopté la culture matérielle et la langue masse s'observent à Bougoudoum. Saavousou et Wima, d'autres ayant adopté la langue et la culture toupouri dans le canton de Doukoula et Tchati-bali, d'autres encore plus au Sud ayant totalement adopté la culture et la langue mousey.

Ainsi donc, l'histoire du Cameroun septentrional porte les stigmates des soubresauts des «Etats» de la cuvette tchadienne puisque depuis le XIXe siècle, époque de la conversion des Kanembou à l'islam jusqu'à la révolte peul, la zone du Sud-ouest du lac Tchad a été l'objet de bouleversements sociaux, d'eclatements d'ethnies, de brassages, de fusions, de migrations vers les massifs-refuges. Face à ces «Etats» impérialistes, les Mandara et les Kotoko s'étaient islamisés, les Mousgoum, les Massa et les Toupouri se réfugièrent dans les zones inondables du Logone et du Mayo-Kebi; les autres se retirèrent dans la chaîne du Mandara. Toutes ces migrations sont antérieures à la constitution de la puissance peul. Ngaoundéré Northern-Cameroon, in: Peter Geschiere / Piet Konings, op. cit. (note 24), pp. 276277.

40

Cité par I. de Carne, op. cit. (note 26), p. 173; voir Beauvilain, op. cit. (note 31), p. 16. 


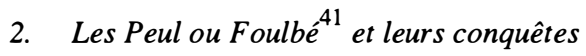

«Toute étude sur les populations du Cameroun», affirme Aaron Su Neba, «notamment celle des régions centrales et septentrionales doit tenir compte, ne serait ce qu'en partie des Peul. La pression qu'ils ont exercée dans le Nord a entraîné de vagues de mouvements qui ont affecté jusqu'à certaines régions du Sud du Cameroun» ${ }^{42}$. Ils sont originaires de la région sénégalo-mauritanienne en Afrique de l'Ouest. Ils ont amené avec eux au Cameroun la foi musulmane. On les retrouve non seulement dans ce pays, mais également au Sénégal, au Mali, en Mauritanie, en Guinée, en Sierra Leone, au Burkina Faso, au Niger, au Tchad et au Soudan, leur système politique est caractérisé par l'existence de chefs très puissants appelés lamibé (lamido au singulier) dont certains avaient défrayé la chronique en s'opposant au régime du Président Ahidjo.

La pénétration peul commence au XVIIIe siècle, bien avant la colonisation européenne au Cameroun et leur histoire est connue: s'infiltrant par familles, poussant devant eux leurs troupeaux, ils payent d'abord tributs aux chefs païens, puis se renforcent peu à peu. Toute la plaine qui borde les Monts Mandara à l'est, à l'ouest et au sud-est déjà ainsi occupée par les pasteurs peul quant au début du XIXe siècle Ousman Dan Fodio proclame la guerre sainte (Jihad) contre l'infidélité environnant. Le Diamaré est soumis en 1808, la Vallée de la Bénoué est largement contrôlée en $1812 .^{43}$

A la même époque, les Mofou et les Guiziga sont chassés de Marwa leur capitale et se réfugient dans les massifs matakam, ce qui provoque des luttes sévères: talonnés de leur côté par les peul qui voulaient s'imposer à l'Ouest. Les Mofou affluent sur les pentes des Monts Mandara après la prise de Goudour. Les Matakam soumis à une triple pression du sud, de l'est, et de l'ouest se regroupent au centre dans les massifs compris entre les vallées

Différentes administrations coloniales sont à l'origine des appellations génériques en usage au Cameroun. Le terme «Peul» a été emprunté par les Français au wolof et son emploi est étendu à d'autres territoires où les Peul étaient présents sans que le wolof y soit parlé. La tendance actuelle est de réserver à ce terme une acceptation d'ensemble qui embrasse tous les Peul, aussi bien ceux qui vivent dans les villages que ceux de la brousse.

Le terme «Foulbé» a été repris par les Allemands aux Peul du Cameroun qui s'appellent ainsi collectivement. Exemple: Pullo, pluriel, Foulbé. Il s'applique aujourd'hui plus précisément aux Peul villageois.

Enfin le terme «Foulani» utilisé par les Anglais provient du Haoussa. Il désigne les Peul dans l'ancien Cameroun Occidental.

R. Dognin, L'installation de Djafoum dans l'Adamaoua camerounais. La Djakka chez les Peul de l'Adamaoua, in: Tardits, op. cit. (note 26), p. 138.

Su Neba, op. cit. (note 7), p. 59.

43 Sur la pénétration peul au Cameroun, voir Eldridge Mohammadou, L'histoire des Peuls ferôbé du Diamaré, Maroua et Petté, Institute for the Study of Languages and Cultures of Asia and Africa (I.L.C.A.A.), 1976, Japan, p. 2. 
de la Tsanaga et du Kérawa et la plaine de Mora. Au Sud, Matakam et Mofu s'allient après des débuts difficiles pour former le groupe «Boulahay». A l'Ouest, aux abords de Madagali, des Marghi se mêlent aux Matakam. mais la majorité forme le bloc assez homogène dit «Mafa» au centre et à l'Ouest du pays autour de Roua et de Soulédé.

Eldrige Mohahhadou, à la suite de nombreuses enquêtes, a pu regrouper les Peul du NordCameroun en trois factions: les Hillaga auxquels il es attribué une vocation guerrière, les Férôbé, éleveurs et habiles politiciens et les Wolarbé plus particulièrement versés dans la connaissance de saintes écritures.

\section{Les Arabes Choa}

Ils sont arrivés au Cameroun il y a plus de trois cent ans du haut Soudan. Ils sont d'origine Hamite et vivent pour la plupart autour du Lac Tchad et dans l'Extrême-Nord. Ils ont un teint clair et se marient souvent avec leurs voisins Kotoko avec qui la collaboration n'est jamais de mise puisque marquée d'incessants conflits en vue de l'espace territorial, ravivés aujourd'hui par les querelles politiciennes et nourris par la psychose des «coupeurs de route» dont les Arabes sont accusés à tort ou à raison d'être les principaux mandants. En outre ils avaient été exclus par le régime Ahidjo du bloc hégémonique régional musulman.

\section{B. L'hégémonie musulmane au Nord-Cameroun}

Avancé par Gransci, le concept d'hégémonie indique une situation historique dans laquelle la domination de classe est fondée non seulement sur la force et la violence mais aussi sur le consentement des classes dominées. Contrôlant l'ensemble des appareils idéologiques dispersés à travers la société civile, la classe hégémonique a la possibilité de diffuser et d'imposer ses représentations, ses valeurs parmi toutes les classes sociales et elle exerce ainsi une fonction de direction idéologique de la société. La réalisation de l'hégémonie assure la cohésion du bloc historique et révèle l'aptitude de la classe dominante à prendre en charge la société tout entière, sur le double plan matériel et spirituel. ${ }^{44}$

L'analyse gramscienne conduit également à poser le problème de la domination idéologique sous un éclairage nouveau. Pour Gramsci, l'hégémonie n'est pas un phénomène figé et stable, elle se conquiert, elle se gagne mais aussi elle se perd.

44

Chevallier, J.J. / Loschack, D., Science administrative, Paris, 1978, tome 1, p. 349; voir R.G. Schwartzenberg, Sociologie politique, Paris, 1988, 4e édition, p. 72. 
L'hégémonie musulmane fondée sur la force et la violence puisque les rapports KirdiMusulmans ont rarement été des rapports de bon voisinage mais davantage des rapports d'oppression des seconds sur les premiers, ignorant la dimension essentielle qui est le consentement n'a jamais été acceptée par les Kirdi et n'a pas résistée après la chute d'Ahidjo.

\section{Les formes d'expression de l'hégémonie musulmane}

Elles concernent l'espace territorial, l'esclavage et l'islamisation voire la «foulbéisation» des Kirdi. Cette hégémonie a été confortée et entretenue par la régime du Président Ahidjo.

\section{a. L'occupation de l'espace territorial par les chefs musulmans}

La prise de pouvoir par les Peul au XIXe siècle concerne la totalité de l'espace territorial régional, que ce soit par les aires qu'ils occupent directement ou que ce soit par les aires qui subissent le contrecoup de cette occupation. Toutefois, ils ne purent s'installer comme nous l'avons dit dans la pointe septentrionale du Nord-Cameroun en raison de la présence des Kotoko et des Mandara, peuples convertis à l'islam respectivement à la fin du XVIe siècle et vers 1715. Les Peul ont donc dû se tailler un territoire plus au sud où leur appartenance à l'islam, n'empêchera pas guerres contre les Mandara. Seulement, quelques groupements peul, sous l'appellation des «Fellata», se maintiennent à proximité du Lac Tchad en s'assimilant aux Arabes Choa.

Les Peul dans la plaine, les autres ethnies (animistes) dans leurs refuges de rochers, ces positions seront maintenues jusqu'à l'arrivée des Allemands qui, en instituant le système d'administration des païens par l'intermédiaire des Peul, officialisèrent et par là-même renforcèrent la domination peul. Il fallut attendre 1924 en plaine et 1940 en montagne, pour que les Français instaurent le système d'administration directe et relèvent les animistes de la tutelle peul.

Cela dit, les terres les plus fertiles et les plus vastes sont occupées par les Foulbé qui conservent leur système politique féodal. Les Kirdi qui se sont réfugiés dans les massifs ou ceux qui s'y sont confinés, ont pu généralement mieux que les gens de la plaine résister à la pression des Foulbé, ils se sont par contre condamnés à vivre sur des terres ingrates, avec des densités de population particulièrement élevées pour des régions défavorables (78 habitants par $\mathrm{km}^{2}$ en moyenne chez les Matakam installés dans les montagnes autour de 
Mokolo, plus de 180 dans certains secteurs, 173 en moyenne dans le canton de Mada, 204 chez les Podoko) ${ }^{47}$ et ces chiffres datent!

Dans la numéro 1 du journal Le Kirdi, Etienne Temwa est clair dans ses propos:

«La situation sociale des Kirdi est préoccupante à plus d'un titre. Des territoires arides et exigus ne nourrissent pas leurs hommes. Est-ce la fatalité?

A l'approche de la saison sèche, on s'inquiète. On se demande si le choléra ou la méningite ne viendront pas s'abattre sur soi ou sur sa famille.

La saison sèche passée, c'est la famine qu'on redoute désormais. C'est cet état d'incertitude qui rythme la vie en milieu Kirdi.»

Dans une grille d'analyse marxiste, Akam Motazé ${ }^{48}$ observe le monde rural du Nord-Cameroun et constate que l'organisation économique d'un lamidat depuis l'Adamaoua jusqu'à la plaine de Mora, montre l'existence de ce que Samir Amin appelle «le mode de production tributaire« et Godelier «une forme africaine de mode de production asiatique», c'est-à-dire un système de prestations. Cela concerne aussi bien les lamidats peul que ceux des Kirdi islamisés de la plaine de Kaélé et de Guidiguis.

Reprenant les analyses de J.C. Froelich ${ }^{49}$, il estime que l'instance économique se résume ici au système de la Djakka, terme peul dérivé du mot arabo-juif Zakkât qui, à l'origine renvoit à une purification et à une aumône légale qui avec le Pèlerinage, la prière, le jeûne et la profession de foi font les cinq piliers de l'islam. En principe le Coran consacre la Zakkât comme une aide aux indigents, aux serviteurs, aux fervents musulmans, aux pèlerins allant à la Mecque etc... Seulement, c'est le lamido gestionnaire du Baïtal (trésor public) qui en est le dépositaire. Et, la Zakkât s'est muée en une redevance que le paysan-sujet paie au lamido. Finalement la Zakkât est devenue un moyen de production et d'accumulation dont s'en servent les lamibés pour assurer leur domination.

Ainsi par exemple, les lamibés utilisent la main-d'oevre de leurs sujets dans leurs champs de coton et perçoivent des dîmes alimentaires allant des paniers de mil aux sacs de ceux qui utilisent leurs «terres». Lors de la fête des moutons, ces chefs exigent un mouton de chacun de leurs sujets.

47

48

49 Yaoundé, 1984, pp. 8 et SS.

Froelich, J.C., Le commandement et l'organisation sociale chez les Foulbé de l'Adamaoua (Cameroun), IFAN, Etudes camerounaises, sept.-déc. 1954, pp. 47 et SS. 
En ce sens, Jean François Bayart ${ }^{50}$ est fondé lorsqu'il voit dans le conflit qui a opposé le lamido de Rey-Bouba aux cultivateurs en 1984 un conflit social et non ethnique encore moins religieux. Il mettait en jeu les rapport de production, traduisant l'exaspération d'une paysannerie surexploitée envers un lamidat et des chefferies de canton par trop prédatrices. Les lettres de protestations rédigées par des fidèles catholiques étaient à cet égard dénuées d'ambiguiité et n'avaient recours aux identifications ethniques qu'à seule fin de qualifier les lignes d'extorsions économiques (A. Touré). On est pas loin des rapports esclavagistes.

\section{b. Les rapports musulmans-Kirdi: le Sceau de l'esclavage}

Dans le Nord-Cameroun, il est difficile d'affirmer ainsi que le note Motazé Akam $^{51}$ que l'esclavage existe ou n'existe pas. Cela naît du fait que les rapports esclavagistes sont observables dans les Saarés (concessions) des lamibés où l'accès à un étranger relève très souvent d'une gageure. D'ailleurs le terme kirdi est péjoratif et associé à la notion d'esclavage. Il permet aux musulmans d'exprimer leur supériorité aux non-musulmans.

L'esclavage a ici deux sources: la naissance dans la servitude et la capture par les razzias qui renvoient à la conquête peul et aux campagnes esclavagistes du XIXe siècle. Ces rapports de production esclavagistes existent dans les milieux fortement islamisés: Dumru, Mora, Guidiguis voir dans les milieux païens esclavagistes comme Kaélé. L'esclave se reconnaît dans le Nord-Cameroun par le fait qu'il ne manifeste aucun désir de vivre de façon autonome. Il participe directement à plusieurs activités agro-pastorales et assume plusieurs tâches économiques dans le Saaré de son maître. Il contribue ainsi à l'accroissement des capacités de production et par conséquent à l'accumulation inégale où le segment musulman sort nanti et riche.

\section{c. L'islamisation voire la "foulbéisation» des populations kirdi}

Les identités religieuses ont tendance à s'ériger en système de classification du champ politique et la norme religieuse fait figure aux yeux de nombreux acteurs sociaux de la loi de référence. Comme au Nord-Nigéria ${ }^{52}$, l'islam a incontestablement façonné au NordCameroun un stock culturel qui donne forme et substance à l'action politique. En effet, pour être légitime, comprise, l'action politique doit être en phase même si cela est de l'«idéologie» avec «ce répertoire de gestes, de symboles, de formes esthétiques, de valeurs cosmiques, religieuses ou morales, de renvois historiques» dont parle J.F. Bayart. C'est dire 
que l'hégémonie passe par une «négociation culturelle» qui lui permet d'avoir des ressources dans la société civile.

Or, l'islam au Nord-Cameroun est ou a été un élément de cette négociation culturelle. Il a pendant longtemps structuré la vie quotidienne, servi de modèle et de grille de lecture, des événements et situations. Il a été donc un système d'explication et de légitimation. Seulement, ici, islamiser signifie également «foulbéiser» c'est-à-dire adopter le modèle culturel peul, la conversion apparaissant comme un moyen sûr de s'intégrer dans la société d'adoption (changement de nom, rejet des siens, refus désormais de parler sa langue maternelle au profit de la langue peul le foufouldé).

L'exemple des femmes Mafa islamisées à Mokolo, ville des Monts Mandara, est assez édifiant: devenues musulmanes, elles changent leur mode de vie. Un trait de la «foulbéisation» est le système complexe d'échanges de mariage. Chez les Mafa, la dot est l'affaire des hommes, mais chez les Foulbé, l'acquisition des cadeaux de mariage est presque entièrement le devoir des femmes. Ainsi la femme mafa, en adoptant l'islam, est confrontée à un problème nouveau. Elle se retrouve dans l'obligation d'accumuler du capital d'une façon ou d'une autre afin de participer aux échanges lors des mariages. Ce qui souvent leur ouvre la porte à la contrebande avec le Nigéria. ${ }^{53}$ Jean Pierre Magnant observe le même phénomène d'oppression des non-musulmans par les musulmans du Tchad voisin. ${ }^{54}$

Dans la monde paysan, l'islam conforte le système lamidal puisque chef religieux, le lamido est le représentant d'Allah sur terre. La relation imaginaire tissant le sujet-paysan à Allah se concrétise donc clairement par une relation idéologique le liant au lamido. L'idéologie islamique détermine à ce titre les liens de dépendance entre le paysan et le lamido et légitime par conséquent le système lamidal. Il paraît normal que le paysan paye les redevances et autres formes d'aumône au lamido à l'instar de la Djakka évoquée plus haut.

L'influence islamique se fit aussi sentir dans le domaine des symboles publics: noms musulmans donnés aux quartiers, interdictions de commercialiser la viande du porc et de l'alcool dans les marchés locaux, construction des mosquées, nomination des Alkali qui rendaient la justice exclusivement selon la sharia.

Et bien que le Président Ahidjo ait assuré son ascension contre le gré des principaux lamibé de la région, qu'il leur eût imposé la création d'un parti politique de conception occidentale,

53 Voir José van Santen, Dot, commerce et contrebande: stratégies d'accumulation chez les femmes «islamisées» de Mokolo, in: Peter Geschiere / Piet Konings, op. cit. (note 24).

54 J.P. Magnant, Tchad: crise de l'Etat ou crise de gouvernement", in: J.F. Medard, Etats d'Afrique noire: formation, mécanismes, crise, Paris, 1991, p. 187. 
qu'il eût restreint leurs prérogatives, il a poursuivi cette stratégie de domination sur l'élément kirdi jusqu'au moment de sa démission en $19822^{55}$

\section{d. La politique d'exclusion, de ségrégation et de marginalisation des populations kirdi par le régime Ahidjo (1958-1982)}

Du temps du régime Ahidjo, l'Etat favorisait systématiquement les musulmans au NordCameroun: les Peul, les Madara, les Kotoko, les Bornouan, les Haoussa et les Kirdi islamisés. Seuls en étaient exclus les Arabes Choa. C'est du moins ce que croient fermement la plupart des non-musulmans dans cette région. Le mémorandum des $\mathrm{Kirdi}^{57}$ est formel làdessus:

\section{«III. La tyrannie d'Ahidjo sur le plan régional}

1. Le commandement

Le tout-puissant Gouverneur de la très grande province du Nord, 99,99 \% des Préfets, Sous-Préfets et Chefs de District sont musulmans et règnent en patriarches sur une population à très forte majorité Kirdi terrorisée.

Leurs sinistres missions

A. Promouvoir la propagation et la diffusion de l'islam sur l'ensemble du GrandNord [...]

B. Etouffer dans l'oeuf toute velléité visant à promouvoir les valeurs culturelles traditionnelles [...]

C. Protéger et étendre les intérêts économiques des Musulmans dans tout le Nord [...]

D. Exploiter au maximum la vigueur des populations Kirdi tout en développant le moins possible leurs régions [...]

E. Retarder au maximum la scolarisation des populations Kirdi [...]

F. Une politique sanitaire inadaptée aux réalités démographiques [...]

G. Canaliser la police $[\ldots]$

Bayart, J.F., L'Etat en Afrique, op. cit. (note 22), p.. 70.

56

Cette politique d'exclusion est contraire au «microdosage» du Président Ahidjo dont parle Pierre Flambeau Ngayap qui «se superposait aux macroéquilibres géopolitiques (au niveau des Provinces) et permettait de mieux chevaucher les réalités socio-politiques que sont les ethnies". Lire Ngayap, P.F., Cameroun. Qui gouverne? De Ahid jo à Biya, l'héritage et l'enjeu, Paris, 1983.

Le Mémorandum des Kirdi sur «l'exclusion de la majorité kirdi» in La Caravane ${ }^{\circ} 12$ du 31 oct. 1991 ou dans le Collectif «Changer le Cameroun»: Le Cameroun éclate? Une anthologie des revendications ethniques, Yaoundé, 1992. 


\section{La même tyrannie sur le plan national}

Ahidjo a voulu présenter un Nord-Cameroun totalement intégré jusqu'à en faire croire à une race.»

Ainsi les termes 〈Haoussa〉, 〈Maïguida〉, 〈Wadjax〉 etc... sont couramment employés par les ressortissants 〈Sudistes〉 pour désigner l'ensemble des populations de cette partie du pays, tant et si bien qu'aujourd'hui on présente le terme «Nordiste` comme une ethnie ou un groupe ethnique, tout comme Bamiléké, Béti, Couala, Bassa etc...

A - Dans le commandement territorial

Tous les ministres Nordistes (ils étaient nombreux et Peul pour plupart) sont musulmans à l'exception de deux ou trois Kirdi ...»

Et la liste est loin d'être exhaustive.

Ainsi que l'affirme Achille Mbembe ${ }^{58}$, une vague d'islamisation (volontaire dans certains, forcée dans d'autres) a agité la région dans les années 70. Bien que cette règle n'ait jamais fait l'objet d'une codification écrite, l'islam a souvent été utilisé comme facteur de discrimination au Nord-Cameroun sous le régime de M. Ahidjo. Dans les faits, les musulmans avaient la priorité en ce qui concernait l'admission dans les concours administratifs, l'attribution des grades dans l'armée, les nominations dans les structures de commandement et les sociétés para-publiques, l'octroi des bourses etc...

A l'origine de cette politique de ségrégation et d'exclusion, d'abord le «paganisme» des Kirdi, leur opposition aux Peul depuis les conquêtes du XIXe siècle et leurs comportements «primitifs» à l'inverse des cultures plus «évoluées» des Peul, des Arabes, des Kanouri et des Haoussa. Traits caractéristiques, «ils se méfient des étrangers, sont agressifs, n'ont pas confiance dans la culture musulmane de leurs suzerains traditionnels, les Peul. Nombre d'entre eux ont adopté certains aspects de la culture peul, arabe et kanouri, tels que les vêtements, mais la plupart restent attaché à la sémi-nudité, aux méthodes agricoles les plus primitives et à l'économie de troc.» Mais avec les changements de structures des sociétés camerounaises (jeunesse, urbanisation, scolarisation, communication, crise économique, ouverture démocratique etc...), ces traits sont en train de s'estomper, avec en prime, la descente massive des jeunes montagnards dans les principaux centres urbains et la part grandissante qu'ils prennent dans le système de l'économie dite informelle; la crise écologique et celle du nomadisme, l'accélération des courants migratoires qui travaillent la sousrégion depuis plus d'une décennie, les phénomènes de saturation foncière, la satellisation croissante de l'économie régionale par le Nord du Nigéria et son éventrement par les inter- 
minables guerres du Tchad sans oublier les églises chrétiennes dont on connaît l'engagement avec les paysanneries non-musulmanes du Nord-Cameroun. ${ }^{59}$

Ensuite, cette politique de marginalisation visait surtout à susciter l'apathie politique des Kirdi. En fait, l'importance politique de la forte population kirdi dans le Nord-Cameroun inquiétait le régime Ahidjo et la colonie musulmane. Car. l'on admet de façon générale que si les Kirdi exerçaient le droit de vote dont ils jouissaient, la domination Peul dans les fonctions électives risquait d'être sérieusement inquiétée. La possibilité leur fut offerte en 1959 lors du plébiscite du Nord-Cameroun britannique, dont les résultats (rejet de l'association avec le Nigéria du Nord après l'accession du Nigéria à l'indépendance en octobre 1960), ne pouvaient être expliqués que par le vote des électeurs kirdi qui avaient exercé leur droit. Apparemment, un nombre suffisant de Kirdi avaient voté contre l'association (pensant peut-être qu'une association continue avec le Nigéria du Nord devait entraîner une prolongation de la domination peul) pour rejeter l'incorporation proposée. Il est significatif d'observer que c'est lors du plébiscite de 1959, au Nord-Cameroun britannique que les Kirdi avaient voté pour la première fois en nombre aussi élevé. Entre les plébiscites de 1959 et de février 1961, le gouvernement du Nigéria avait mené une campagne politique d'envergure, leur lourde artillerie visant particulièrement les Kirdi. Cette stratégie avait porté ses fruits et lors du plébiscite de 1961, ce furent toujours les Kirdi qui avaient fait pencher la balance électorale en faveur de l'intégration avec le Nigéria. Ce potentiel politique des Kirdi faisait de leur éveil un sujet de préoccupations considérables pour le régime Ahidjo. $^{60}$

Néanmoins cette hégémonie d'a jamais été intériorisée par les Kirdi et est aujourd'hui battue en brèche.

\section{Les limites et la crise de l'hégémonie musulmane}

Réduire les rapports musulmans-Kirdi aux simples rapports dominants-dominés reste une illusion si l'on ne prend en compte la capacité de ces derniers à l'indocilité. ${ }^{61}$ En fait l'hégémonie peul sur la multitude des ethnies autochtones est restée instable et limitée: jusqu'aujourd'hui, certains groupes Kirdi ont réussi à se soustraire au pouvoir politique et économique des Peul comme par exemple ceux qui peuplent le sud de la Diamaré, dans l'actuelle province de l'Extrême-Nord (région de Kaélé): les Toupouri, les Moundang et les Guiziga. Encore que pendant les guerres de conquête du XIXe siècle, les Peul (le lamido de

59

60

61

Mbembe, A., op. cit. (note 24), pp. 352-353.

Voir Le Vine, V.T., op. cit. (note 6), p. 124.

L'expression est empruntée à Achille Mbembe, Afriques indociles: christianisme, pouvoir et Etat en société post-coloniale, Paris, 1988. 
Kalfou) avaient été vaincus par les Toupouri trop nombreux et sans pouvoir centralisé. Même avec l'aide des Allemands qui avaient introduit le gouvernement indirect, les Peul n'avaient pas réussi à établir l'ordre et la sécurité dans la région. L'administration française qui remplaçait les Allemands après la première guerre mondiale s'était alors vue contrainte de grouper ces Kirdi en cantons «indépendants». 62

En témoigne cette lettre de M. Fourneau, adressée en 1918 au chef de circonscription de Maroua:

«Je voudrais que la question des commandements Kirdi soit réglée une fois pour toutes. L'antagonisme entre les deux races n'est pas à démontrer. Il n'apparaît pas plus difficile - pour ne pas dire moins - de mettre les Kirdi sous notre autorité directe que de les placer sous celle des sultans [...]

Ne compliquons pas inutilement notre tâche en voulant plier sous le joug foulbé des populations qui ont préféré vivre au milieu des terres ingrates que de se soumettre à eux.r 63

Les accrochages entre les Kirdi et les Foulbé étaient monnaie courante: la supériorité militaire des Foulbé permettait rarement des batailles de type classique: conscients de leur faiblesse, les Kirdi organisaient surtout des réseaux d'actions nocturnes qui leur permettaient notamment de dérober du bétail. Certains chefs Kirdi (le chef guiziga de Lulu et le Masaï de Muda par exemple) furent destitués respectivement en 1929 et 1937 . L'administration française reprochait à l'un de multiples larcins, à l'autre une attitude trop compréhensive vis-à-vis des voleurs. ${ }^{64}$

S'agissant de l'islamisation, elle est et fut limitée. Ne touchant très souvent que les segments sensibles de l'épiderme social tels que les chefs traditionnels et surtout sans véritable emprise sur le monde rural. D'ailleurs le Kirdi s'islamisait en vue d'une promotion sociale, donc pour des gains matériels et personnels. Et dans ce contexte il continuait à pratiquer sa religion «traditionnelle». En outre les lamibé islamisés étaient ambigus: le lamido de Doubane, un Toupouri, s'était islamisé mais ne jeûnait pas pendant le Ramadan. C'était le même son den cloche frondeur et réfractaire chez les lamibés de Boboyo et de Moutouroua. ${ }^{65}$

Pour Kees Schilder, cette islamisation limitée trouve son explication surtout dans le fait que le pouvoir étatique régional sur lequel se fondait l'hégémonie peul était également limité.

62

63

64

65

Voir Kees Schilder, Etat et islamisation..., op. cit. (note 19), p. 144; voir également M.J.P. Fogui, op. cit. (note 4), pp. 58-59.

Rapporté par Guy Pontié, op. cit. (note 25), p. 40.

Ibid., p. 44.

Akam Motazé, Contribution à l'étude sociologique du monde rural..., op. cit. (note 48), p. 12. 
Les structures administratives dans le Nord étaient extrêmement légères, surtout au début de la période post-coloniale. Et de ce fait, les chefs locaux ont pu garder une marge de manoeuvre politique appréciable. D'autre part, l'islamisation des populations rurales dépendait de la coopération active de ces chefs qui n'étaient par conséquent pas des autorités religieuses. De plus, toutes ces chefferies n'étaient pas bien intégrées aux sociétés locales kirdi. En dernier lieu, le Cameroun étant constitutionnellement un Etat laïc, la promotion de l'islam par les fonctionnaires était en principe illégale, de ce fait. il était difficile de réaliser une campagne organisée et efficace; les moyens matériels et financiers étaient probablement limités. ${ }^{66}$

Le pouvoir étatique étant limité sur le plan régional, les populations rurales disposaient d'une autonomie considérable au niveau local, ce qui a donné naissance à des modes populaires d'action (a-)politique contre la fièvre «missionnaire» d'en haut. L'islamisation était nominale. On peut douter fortement que le simple rituel de conversion soit un indicateur adéquat de l'islamisation, la conversion étant souvent plus l'expression d'une allégeance politique qu'un acte religieux. La plupart des nouveaux convertis non seulement continuèrent leurs activités religieuses «traditionnelles» mais aussi à consommer autant de viande de porc et de bière de miel qu'avant. Dans beaucoup de cas, on n'adopta que les symboles islamiques les plus voyants, tels le boubou et le Ramadan. Ce n'est donc pas surprenant qu'une déislamisation se soit produite au cours des années 1980 , quand l'hégémonie musulmane s'est trouvée affaiblie à cause de la succession présidentielle de 1982 et du coup d'Etat avorté à Yaoundé en 1984. Dans les villages comme Boboyo, observe Kees Shilder, on ne trouve plus guère de musulmanes aujourd'hui. ${ }^{67}$

En outre, la christianisation a parfois été une manière de protester contre le joug musulman.

On ne peut pas cependant conclure de ce qui précède que l'établissement de l'hégémonie peul sur les sociétés kirdi ait été un échec. D'abord, les Peul demeurent économiquement puissants au Nord-Cameroun et, tant que le pouvoir économique des Kirdi sera précaire, ils ne pourraient prétendre jouer un véritable contrepoids au segment socialement dominant musulman. Il ne peut avoir d'émancipation politique sans un minimum d'autonomie et de garanties économiques. Néanmoins, comme en Afrique c'est la référence à l'Etat qui conduit à l'accumulation, puisqu'il faut être riche pour avoir de l'influence et l'influence permet de s'enrichir, le réveil et l'intégration politique des Kirdi leur permettront et leur permettent déjà de contrôler les ressources rares de l'Etat camerounais. Ensuite, l'islamisation visait moins de convertir que de créer à travers les chefs locaux des alliances politiques dans le

Kees Schilder, Etat et islamisation..., op. cit. (note 19), pp. 146-147.

67 Ibid., p. 147. 
but de perpétuer l'apathie politique des Kirdi et de sauvegarder le mirage de l'unité politique et sociologique du Nord.

Par ailleurs, grâce au système des lamidats et aux divers mécanismes de contrôle et d'assujettissement hérités du XIXe siècle et renforcés sous la colonisation allemande et française, puis sous le régime du Président Ahidjo, le pouvoir peul dispose de solides relais au sein des populations Kirdi. Il y maintient son emprise à travers un tissu de relations clientélaires (dîmes et impôts divers, formes diverses de servage, contrôle des terres et des pâturages, de l'élevage et de l'artisanat, quasi-monopole des réseaux de la contrebande avec le Nigéria et le Tchad et du commerce des tissus, voitures, des radio-cassettes et des produits industriels revendus sur le marché local). Dans la plupart de cas, ces relations clintélaires et la structure de domination dont elles sont l'expression sont plus décisives que le sentiment d'appartenance ethnique. Les réseaux d'obligations et de réciprocité dans lesquels ces relations enserrent les paysans Kirdi pèsent au niveau local, d'un poids particulier sur la définition des appartenances politiques et sur le comportement électoral. ${ }^{68}$

\section{Accumulation et politisation des groupes ethniques au Nord-Cameroun}

En Afrique noire contemporaine, antérieur au fait étatique existe le fait ethnique. Paradoxalement celui-ci trouve dans celui-là un terrain privilégié de résurgence et d'expression. C'est à tort que l'on prétendrait minimiser la vivacité importante de cette donnée sociologique. De l'Afrique, il explique certains comportements auxquels s'intéresse la science politique. L'Africain est compris entre deux faits qui luttent pour la suprématie. L'observation de M. Nicolas s'impose ici avec force lorsqu'il écrit: «Si le fait ethnique n'était qu'une trace du passé, on ne comprendrait cette obstination de ceux qui visent à l'adapter sans cesse au présent le plus brûlant, ni qu'il provoque la mise en cause des systèmes modernes» ${ }^{69}$.

C'est qu'on ne peut pas parler de structure sociale en Afrique et singulièrement au sud du Sahara sans évoquer le tribalisme qui se pose partout en trouble-fête, mettant à mal les schémas idéologiques les plus élaborés. Ce qui a fait dire à Lanciné Sylla que: «Tous les Etats africains actuels sont des Etats volcans ... car ce qui fait battre le coeur des jeunes nations africaines, c'est la conjoncture du tribalisme et du parti unique».

En fait le tribalisme revêt en Afrique la forme d'une religion: on est tribaliste comme on est catholique ou musulman avec ce que cela comporte d'illusion grégaire ou de répulsions subjectives vis-à-vis des membres des autres groupes. Mais alors qu'être catholique ou

Mbembe, A., Crise de légitimité..., op. cit. (note 24), pp. 353-354.

69

Voir Pambou Tchivounda, Essai sur l'Etat africain post-colonial, Paris, 1982, p. 92. 
protestant n'empêche pas d'avoir des opinions divergentes sur différents sujets, être tribaliste en Afrique s'apparente à une filiation définitive, à une adhésion sans rémission, à tout ce que les systèmes politiques mis en place sont exclusifs (on est soit à l'intérieur, soit en dehors ou on est avec moi ou l'on est contre moi). ${ }^{70}$

Le Cameroun pour sa part n'échappe pas à cette analyse où aujourd'hui on assiste à un regain de tribalisme. ${ }^{71}$ La conclusion de $\mathrm{M}$. Prouzet est encore d'actualité: «La prééminence du facteur ethnique sur le facteur idéologique apparaît clairement dans la vie politique des citoyens. C'est ce qui ressort en premier lieu d'un examen portant sur la situation des partis politiques au Cameroun avant la disparition de ces derniers ou avant leur fusion dans l'U.N.C. [...] le ler septembre 1966» ${ }^{72}$.

Un observateur devait d'ailleurs déclarer en ce sens:

«A la différence de la France, la température de la vie politique camerounaise monte ou baisse moins à cause de l'intensité de la lutte purement idéologique qui peut séparer deux formations politiques différentes qu'à cause de ce que l'on pourrait appeler l'esprit de clocher qui les anime.

En tout cas, on peut dire tant sur le plan doctrinal et idéologique que sur le plan de la pratique que la différence est presque nulle d'un bord à l'autre qui sépare les formations politiques de gauche et de droite s'agissant des partis politiques camerounais...

En somme, l'esprit de clocher aggravé, nourri et dominé par l'important problème tribal et les facteurs traditionnels qui en sont le soubassement fait de ces formations dites politiques de petites factions rivales qui s'opposent les unes aux autres moins à cause de la doctrine qui les anime qu'à cause des leaders qui sont à leur tête et qui en sont la personnification.» ${ }^{73}$

Dans une Afrique traditionnelle et rurale peu habituée à véhiculer des idées surtout lorsqu'elles ont été enfantées dans d'autres contextes, on ne vote pas pour une idéologie, une politique, mais pur un homme qui disposera du pouvoir de donner de l'argent, un emploi, des faveurs à toute la famille, à toute son ethnie. Nous sommes arrivés au point où le tribalisme n'a plus rien à voir avec la cohésion tribale d'antan. Dans une société éclatée où chacun lutte pour sa survie, on se raccroche à la seule bouée disponible «le nationalisme

Etounga Manguelé, D., Cent ans d'aliénation, Paris, 1985, p. 233.

71 Voir sur ce point, Valentin Ndi Mbarga, Ruptures et continuités au Cameroun, Paris, 1993, pp. 224 et SS; voir Mongo Beti, La France contre l'Afrique. Retour au Cameroun, Paris, 1993.

73 Zang Atangana, J.M., Les forces politiques au Cameroun réunifié. Les partis politiques avant la réunification, Paris, 1989, tome 1, pp. 71-72. 
ethnique désintégrateur de la nation territoriale», que les dirigeants prétendent vouloir bâtir. Car, si un individu est Président de la République, sa famille ou son clan règne sur sa tribu; et sa tribu règne sur toutes les autres tribus de la nouvelle nation. ${ }^{74}$

Certes, certains leaders, à l'instar de Paul Biya, No John Fru Jdi, Bouba Bello Maïgari, Adamou Ndam Njoya se sont élevés au-dessus des contingences tribales en ce sens que les structures de leurs partis sont présentes dans toutes les provinces du pays, mais tous comptent sur le soutien actif de leurs ethnies ou des habitants de leurs régions d'origine. Nous n'en voulons pour preuve que les législatives du ler Mars 1992: ${ }^{75}$

- L'U.P.C. (Union des Populations du Cameroun) conduite par son Secrétaire Général Augustin Frédéric Kodock de l'ethnie bassa obtint en priorité les suffrages des populations bassa: tous les 3 sièges de la Sanaga Maritime, tous les 3 sièges du Nyong-Ekellé, 5 sièges à Douala où existe une forte colonie bassa et l'unique siège du Nkam chez leurs cousins.

- Le R.D.P.C. (Rassemblement Démocratique du Peuple Camerounais) de M. Paul Biya, se fit d'abord retentir dans les zones beti-boulou des provinces du Centre, Sud et Est en raflant 41 sièges sur les 50 en lice, à commencer par les 5 du Dja-et-Lobo où est né le Président Biya. Il est vrai qu'il n'y avait en compétition que la liste du RDPC, mais le fort taux de participation $(90,71 \%)$ en était révélateur.

- L'UNDP (Union Nationale pour la Démocratie e t le Progrès) du Peul Bouba Bello Maïgari s'est d'abord assurée de tous les 9 sièges de l'Adamaoua, de tous les 12 du Nord et une percée à l'Extrême-Nord avec 10 députés.

- Toujours à l'Extrême-Nord, le MDR (Mouvement Démocratique pour la Défense de la République) du Toupouri Dakolé Daïssala obtint 6 sièges dans les zones toupouri du Mayo-Danaï et du Mayo-Kani.

Si l'ethnicité mise en rapport avec le pouvoir est ainsi un enjeu électoral dans l'échiquier politique camerounais, il paraît nécessaire d'étudier le comportement électoral des populations due Nord-Cameroun après qu'elles aient été pendant longtemps embrigadées dans une logique pseudo-unitaire «ahidjoiste».

\section{A. L'U.C. et la stratégie pseudo-unitaire du Président Ahidjo}

L'U.C. a été créée en 1958 par Ahmadou Ahidjo, peu avant son accession à la primature. Au moment de sa création, ce parti n'échappait pas aux pesanteurs «tribales». Son fondateur 
dont le caractère régional ne faisait aucun doute, comme en témoignaient leurs sigles: ainsi après son élection à l'ARCAM (Assemblée Représentative du Cameroun) en 1946, il avait fondé dès 1948, l'Association Amicale de la Bénoué (du nom de son département). Il se hissa au niveau régional en 1956 en fondant le mouvement politique pour l'évolution du Nord-Cameroun (E.N.C.). ${ }^{76}$

\section{L'U.C. - un parti régional au service du bloc de pouvoir musulman du Nord-Cameroun}

M. Fogui observe que la création de l'U.C. en 1958 représentait une étape importante dans la stratégie du Président Ahidjo: il venait d'être nommé Premier Ministre dans le deuxième gouvernement. Les négociations avec la France et la «rébellion» upéciste exigeaient un chef de gouvernement au pouvoir sûr. Il a donc cherché à rassembler les populations du Nord au sein d'un même parti avant d'aborder les pourparlers avec la classe politique du Sud. C'est cette stratégie unitaire qui a constamment fait défaut aux hommes politiques du Sud empêtrés qu'ils étaient dans les querelles de personnes et/ou tribales. ${ }^{77}$

Ce qui est incontestable, c'est que dès sa création, l'U.C. était un parti régional. Non seulement tous ses élus à l'ALCAM (Assemblée Législative du Cameroun) étaient originaires du Nord, mais le Comité Directeur élu au Congrès Constitutif de Garoua en 1958 ne comprenait aucun Sudiste. Il a fallu attendre le IIe Congrès de Ngaoundéré en 1959 pour voir deux hommes du Sud accéder à ce Comité Directeur (Marigoh Mboua et Mballa Henri).

L'U.C. n'a participé qu'à quelques consultations populaires: les élections législatives du 10 avril 1960, le référendum du 21 février 1961 qui portait sur l'adoption de la constitution, les élections partielles de décembre 1961 et les municipales de décembre 1962. Et lors des législatives de 1962 par exemple, non seulement il n'y avait pas de liste concurrente au Nord-Cameroun mais également le taux de participation était élevé avec un résultat de $95 \%$ grâce au soutien des chefs peuls. ${ }^{78}$

En tout cas, les intentions de M. Ahidjo étaient limpides. Parvenu au pouvoir en 1958 parce qu'il paraissait le mieux à même de conjurer les velléités sécessionnistes de l'aristocratie peul, il a opposé au morcellement politique du «Sud» la construction arbitraire d'un «Nord» unitaire et immense que surveillait un gouverneur inamovible. Cette base arrière n'a cessé de représenter pour lui une ressource politique majeure garante de la permanence de sa

76

77 78

Fogui, J.P., op. cit. (note 4), p. 69.

Ibid., pp. 69-70; voir également J.F. Bayart, L'Etat au Cameroun, op. cit. (note 22), pp. 46 et SS.

Zang-Atangana, J.M., Les forces politiques au Cameroun réunifié: l'expérience de l'U.C. et du K.N.D.P., Paris, 1989, tome 2, p. 51. 
position bien qu'elle fût négative su segment Kirdi. C'est ce faux monolithe que parvenu au pouvoir en 1982, M. Biya s'est employé à démanteler à partir de 1983 en faisant fructifier les frustrations qu'il engendrait. Frustrations de Maroua et Ngaoundéré dont les lamibés avaient été frappés de plein fouet en 1958 et 1963 pour avoir contesté M. Ahidjo et qui s'étaient vus systématiquement préférer Garoua. Colère rentrée de la masse des cultivateurs païens ou christianisés pressurés par une chefferie de canton islamisée et intégrée au bloc social dominant de la région. Déception des missions catholiques sournoisement persécutées par cette chefferie pour s'être intéressées de trop à la condition paysanne. ${ }^{79}$

\section{Mutations socio-politiques de déconstruction de l'hégémonie musulmane}

Au début de la décennie 80, un certain nombre d'événements vont précipiter au Cameroun le lézardement de l'hégémonie musulmane: la démission de $M$. Ahid jo à la tête de d'Etat et la succession présidentielle de M. Biya qui enchantera à jamais les Kirdi le 6 novembre 1982.

Comme le fait remarquer le Professeur Georges Burdeau ${ }^{80}$, un chef est populaire parce qu'il est tenu pour simple, parce qu'il se fait comprendre et parce qu'il libère de ses frustrations le groupe qui le soutient. Et c'est bien parce que la popularité ne dénature pas le pouvoir en le rendant accessible qu'une des voies les plus sûres pour l'atteindre n'est pas de cacher la puissance, mais, au contraire d'en exagérer les manifestations dans le sens que le peuple attend. Et ce qu'il attend, c'est d'abord d'être délivré d'un complexe d'infériorité. Le chef populaire est celui qui dit non. Non au colonisateur, non aux technocrates, non aux puissances économiques, non aux impérialismes, qu'il soient de l'est ou de l'Ouest, non à toutes les forces par lesquelles les individus se sentent opprimés. Ce geste négatif en même temps qu'il fait porter par les «autres» la responsabilité du mal prouve au groupe l'impression que le pouvoir va enfin l'affranchir d'une dépendance qui lui est intolérable.

Parlant de l'avènement du Président Biya, le Kirdi Dakolé Daïssala, courtisan de Biya ${ }^{81}$ et ennemi d'Ahidjo écrit:

«Et la conviction profonde de notre peuple fut qu'il franchissait cette frontière qui sépare la nuit de la lumière.

Burdeau, G., Traité de science politique. Le pouvoir politique, Paris, 1966, tome 1, 2e édition, pp. 448-449.

81 Jeté en prison en 1984 (à la suite du putsch manqué) par le régime Biya comme la plupart des Nordistes, Dakolé fut libéré en 1990. En 1991, il crée le MDR et aux législatives de 1992, son parti gagne 6 voix qui lui permettent de négocier une coalition gouvernementale avec le RDPC d'où il est Ministre d'Etat. 
En cette aube nouvelle où notre histoire reprend sa marche rigoureuse et résolue vers des horizons nouveaux et exaltants, je me trouve à Goundaye, dans mon village natal, en congé annuel.

Je me suis fait le devoir de parcourir des centaines de kilomètres à la seule fin de me mettre au diapason de l'euphorie collective partout présente, à commencer par mon village qui s'est souvenu de ses misères permanentes et de ses démêlés plus ou moins feutrés avec l'ancien régime. Je puis témoigner que dans les secteurs couverts, l'euphorie des populations (majoritairement Kirdi, il est vrai) ne pouvait se composer qu'aux explosions de joie spontanées et émouvantes qui marquent la proclamation de l'indépendance nationale dans une ville reconquise avec quelque chose d'indescriptible en plus.

〈Paul Biya est notre messie`, pouvait-on entendre dans la bouche de ceux qui ne pèsent pas leurs mots ...» ${ }^{82}$

Les rapports entre Biya et son ancien patron vont vite se détériorer et en juin 1983, les services de renseignements découvrent un complot ourdi contre M. Biya visant à assassiner ce dernier avec pour commanditaire M. Ahidjo et pour cerveaux le Capitaine Salatou et le Commandant Ibrahim Oumarou tous deux musulmans, respectivement ancien aide-de-camp et intendant de $\mathrm{M}$. Ahidjo. Il s'en suivra une condamnation à mort de ces derniers (y compris M. Ahidjo) puis commuée en détention à perpétuité. Fort heureusement, ils ont été libérés avec le vent de la décennie 90 .

A la suite de cet incident, le Premier Ministre Bouba Bello Maïgari, un Peul, est limogé et remplacé par un Kirdi, le Toupouri Ayang Luc. Biya divise le Nord en trois privinces (Adamaoua, Nord et Extrême-Nord), ce qui offre aux Kirdi un pôle d'expression de leurs exigences, l'Extrême-Nord où ils sont localisés en majorité. Dès ce moment, l'ancien axe politique Nord-Sud, fruit de l'alliance des aristocraties du Nord et des élites du Sud qui a permis à Ahidjo de gouverner se déplaçait vers l'Extrême-Nord (donc amputé de l'Adamaoua et du Nord administratifs).

Le coup d'Etat avorté du 6 avril 1984 conduit essentiellement par les éléments de l'ancienne garde d'Ahidjo, la Garde Républicaine formée en majorité d'éléments Kirdi (et on avance que c'est l'une des causes de l'échec de cette mutinerie) encadrés par les officiers musulmans à commencer par son chef le Colonel Saleh Ibrahim (exécuté pour la circonstance) amènera définitivement le Président Biya à sceller un pacte avec la majorité Kirdi et briser ainsi l'hégémonie musulmane. 
Dans tous les cas, sous le régime du Président Biya, les musulmans ont perdu beaucoup de leur pouvoir non seulement à Yaoundé mais aussi dans le Nord du pays. L'arrogance musulmane, la répression culturelle et la discrimination ont nettement diminué à tel point que beaucoup de non-musulmans parlent aujourd'hui de «libéralisation» pour désigner ces changements. Et depuis quelques années, l'intérêt des fonctionnaires non-musulmans pour l'histoire et les traditions ancestrales de leurs ethnies va croissant. Cette renaissance culturelle parmi les populations se traduit au niveau de l'initiation «traditionnelle» de leurs enfants et des comités de développement cantonaux et villageois qui sont contrôlés et initiés par l'Administration. Elle prend la forme d'explosion culturelle et de semaines culturelles. 83

En outre, les associations d'entr'aide des fonctionnaires non-musulmans dans les grandes villes du Cameroun sont responsables de la politisation de ce «mouvement culturel». Beaucoup de ces «groupes de ressortissants» en 1991 ont débattu des changements politiques en cours, ce qui a eu comme résultat la création à Yaoundé de la DCK (Dynamique Culturelle Kirdi), une tentative de réunir les élites non-musulmanes du Nord dont l'ancien Ministre du gouvernement Biya, Jean Baptiste Baskouda, est l'un des grands artisans. Les personnalités de la DCK liées au RDPC ont joué un rôle important dans la diffusion des sentiments antimusulmans parmi la population non islamisée du Nord, ce qui a contribué à renforcer la méf iance à l'égard de l'UNDP. ${ }^{84}$

L'intégration du terme Kirdi dans le discours politique illustre bien ce processus: contrairement à ce qui se passait encore il y a quelques années, beaucoup ont aujourd'hui ce mot à la bouche. Les élites non-musulmanes ont adopté et popularisé ce mot, ils en ont fait un terme politique qui reflète une protestation contre l'exclusivisme et la discrimination du côté des musulmans et une prise de conscience croissante que les non-musulmans peuvent représenter une force politique importante s'ils s'unissent. ${ }^{85}$

Autre chose est la création d'unités administratives opérée par le régime Biya dans le NordCameroun. En effet, durant le régime du Président Ahidjo, le Nord qui représente près de $35 \%$ du territoire national était sous-administré avec une province et six départements (Bénoué, Vina, Margui Wandala, Logone-et-Chari, Mayo Danaï et Diamaré) au contraire du Sud qui en avait respectivement six et trente quatre.

Kees Schilder, La démocratie aux champs: les présidentielle d'octobre 1992 au Nord-Cameroun, 84 in: Politique africaine $n^{\circ} 50$, Paris, juin 1993, p. 119. Ibid.

L'ancien Ministre Jean Baptiste Baskouda vient d'écrire un ouvrage dans ce sens: Kirdi est mon nom, Yaoundé, août 1993; le journal Le Kirdi a vu le jour en 1991; le terme kirditude est de plus en plus usité. 
L'un des premiers actes de M. Biya a consisté dès 1983 à diviser le Nord en trois provinces dont l'Adamaoua, ancien département de la Vina avec quatre départements (Vina, Mayo Banyo, Mbéré et Faro-et-Déo); le Nord, ancien départment de la Bénoué avec quatre départements (Bénoué, Faro, Mayo-Rey et Mayo Louti) et l'Extrême-Nord avec six départements (Diamaré, Mayo Sava, Mayo Tsanaga, Mayo Kani, Mayo Danaï et Logone-etChari).

Or, accumulation et enrichissement sont devenus les maîtres mots de l'ultime justification du processus de création de nouvelles unités administratives puisqu'elle signifie traditionnellement l'établissement d'une fonction publique locale, la distribution d'inf rastructures, d'hôpitaux, d'écoles, de logements etc... Et, atteindre de tels objectifs passe généralement par la recherche, voir l'invention de fondements historiques et culturels susceptibles de conférer aux communautés concernées une identité face aux autres. L'appel à l'ethnicité constitue donc pour le pouvoir un moyen de susciter loyauté et mobilisation locales. ${ }^{86}$

Par ailleurs, après la coalition gouvernementale RDPC-MDR et l'appui de Dakolé Daïssala à l'élection du Président Biya en 1992, les Toupouri, à travers le MDR, ont tout d'un coup eu accès au centre du pouvoir politique, ce qui a eu ses avantages. Dakolé a réussi à s'assurer de gains matériels et financiers. Sa maison de Kaélé s'est transformée en luxueuse villa. Il a pu obtenir un reclassement administratif qui a augmenté le nombre de cantons en pays toupouri et massa, ce qui veut dire davantage d'emplois sur place. Des écoles secondaires ont été ouvertes et des maires d'obédience MDR ont remplacé leurs prédécesseurs RDPC à Kaélé et à Guidiguis.

En outre, des Kirdi, membres du MDR se sont vus propulser au devant de la scène politique nationale, dans les hautes instances de l'Etat: Dakolé Daïssala, coordonnateur national du MDR promu au rand de Ministre d'Etat pour la première fois un Ndourou de l'Adamaoua est nommé ministre, M. Pierre Souman, il en est également du Mafa Bava Djingoer, le Gidar Daway Rou (Secrétaire d'Etat) sans oublier le Massa Djona Jean, député et VicePrésident de l'Assemblée Nationale.

On peut multiplier d'autres exemples dans les rangs du RDPC: le Président du Conseil Economique et Social est un Toupouri, M. Luc Ayang, ancien Premier Ministre; Jean Baptiste Baskouda et Tikela Kemone, anciens ministres de Paul Biya et aujourd'hui Conseillers à la Présidence; Zacharie Pérévé, Secrétaire d'Etat. D'ailleurs, le Président de l'Assemblée National, le chef Mada Cavaye Yegué Djibril n'est autre qu'un Kirdi islamisé. Etats d'Afrique noir, op. cit. (note 54), p. 124. 


\section{B. La restauration du multipartisme et revanche des populations Kirdi}

Outre l'émancipation des populations Kirdi opérée par le régime Biya, le multipartisme restauré au Cameroun au crépuscule de l'année 1990 marque un tournant décisif au NordCameroun. Il constitue un progrès considérable qui met un terme à leur apathie politique. Désormais elles vont utiliser comme tribunes des partis politiques pour assurer leur revanche contre le bloc hégémonique musulman. A ces victimes du passé, il convient de signaler les Arabes Choa, musulmans jadis ignorés par le régime Ahidjo. ${ }^{87}$ Ils redoutent un retour au pouvoir des Peul et soutiennent farouchement le RDPC du Président Biya contre leurs frères «ennemis» Kotoko moulés eux dans le sérail de l'UNDP. A ces deux partis, il faut ajouter le MDR de Dakolé Daïssala qui recrute en majorité chez les Toupouri.

\section{Le Nord-Cameroun comme fief traditionnel de l'UNDP}

L'U.N.D.P. a fait ses premiers pas sous l'impulsion de M. Samuel Eboua (au moment où Bouba Bello Maigari, son actuel leader, était en exil) ancien Secrétaire Général à la Présidence sous Ahidjo. Après la brutale éviction de celui-ci en 1991, cette tâche est revenue à M. Bouba Bello Maïgari, ancien Premier Ministre de novembre 1982 à juin 1983. Si l'on s'en tient au nombre de ses députés au parlement (68), l'UNDP est la deuxième formation d'opposition. L'éviction de M. Samuel Eboua lui a cependant coûté des sympathies au SudCameroun. Ce parti dispose néanmoins d'une solide base régionale dans les provinces de l'Adamaoua, du Nord et de l'Extrême-Nord. Toutes les trois totalisent 30,3 \% de la population du pays. La fragilité de son implantation régionale ne doit pourtant pas être perdue de vue. En revendiquant à tue-tête l'héritage de $\mathbf{M}$. Ahidjo, ce parti largement dominé par les Foulbé fait signe d'un passé controversé et au sujet duquel il n'existe pas de consensus au Nord. $^{88}$

Aux législatives de mars 1992, l'UNDP était sortie sans conteste vainqueur des urnes dans les trois provinces septentrionales comme nous avons vu plus haut et comme l'illustrent ces tableaux synoptiques. 89

Les Kotoko qui ont une origine Sao (premiers occupants de la pointe septentrionale du Cameroun) sont minoritaires, intellectuels et se réclament «autochtones» au contraire des Arabes Choa, majoritaires, illettrés, nomades mais riches commerçants que les Kotoko qualifient d'envahisseurs. Ce qui est source de conflits entre les deux communautés nourris par les querelles politiciennes.

Voir A. Mbembe, Crise de légitimité..., op. cit. (note 24), p. 350.

89

Source: Cameroon Tribune $n^{\circ} 5089$, op. cit. (note 75). 
a-La Province de l'Adamaoua

\begin{tabular}{|l|c|c|c|c|}
\hline & \multicolumn{2}{|c|}{ U.N.D.P. } & \multicolumn{2}{c|}{ R.D.P.C. } \\
\hline Départements & $\%$ & Elus & $\%$ & Elus \\
\hline Vina & 81,90 & 3 & 12,36 & 0 \\
\hline Mayo Banyo & 84,46 & 2 & 15,54 & 0 \\
\hline Mbéré & 51,20 & 3 & 42,68 & 0 \\
\hline Faro-et-Déo & 74,91 & 1 & 25,90 & 0 \\
\hline
\end{tabular}

\section{$b$ - La Province du Nord}

\begin{tabular}{|l|c|c|c|c|}
\hline & \multicolumn{2}{|c|}{ U.N.D.P. } & \multicolumn{2}{c|}{ R.D.P.C. } \\
\hline Départements & $\%$ & Elus & $\%$ & Elus \\
\hline Faro & $/$ & 1 & $/$ & 0 \\
\hline Mayo Louti & $/$ & 4 & $/$ & 0 \\
\hline Mayo-Rey & $/$ & 3 & $/$ & 0 \\
\hline Bénoué & $/$ & 4 & $/$ & 0 \\
\hline
\end{tabular}

c-La Province de l'Extrême-Nord

\begin{tabular}{|l|c|c|c|c|c|c|}
\hline & \multicolumn{2}{|c|}{ U.N.D.P. } & \multicolumn{2}{c|}{ R.D.P.C. } & \multicolumn{2}{c|}{ M.D.R. } \\
\hline Départements & $\%$ & Elus & $\%$ & Elus & $\%$ & Elus \\
\hline Mayo Sava & 42,96 & 0 & 57,04 & 4 & $/$ & $/$ \\
\hline Mayo Tsanaga & 31,19 & 3 & 49,74 & 3 & $/$ & $/$ \\
\hline Mayo Kani & 33,94 & 3 & 19,89 & 0 & 42,90 & 3 \\
\hline Mayo Danaï & 33,22 & 1 & 21,50 & 1 & 39,28 & 3 \\
\hline Logone-et-Chari & 43,65 & 0 & 56,35 & 4 & $/$ & $/$ \\
\hline Diamaré & 64,60 & 5 & 26,43 & 0 & 4,00 & 0 \\
\hline
\end{tabular}


Lors de l'élection présidentielle, le score de Bello fut nettement moins glorieux dans les trois provinces, il obtint moins de la moitié des voix. Soit $64,04 \%$ dans lAdamaoua, $50,42 \%$ dans le Nord et $42,88 \%$ à l'Extrême-Nord. ${ }^{90}$ Un recul qui s'explique surtout par la popularité grandissante du RDPC dans la province de l'Extrême-Nord, l'une des plus grandes du pays avec $17 \%$ du total des électeurs inscrits.

Aujourd'hui, l'UNDP est minée par des querelles intestines entretenues par le RDPC du Président Biya: à l'origine, les élections législatives où l'UNDP rafle soixante huit sièges et devient le deuxième poids lourd du parlement. Après l'élection présidentielle, et malgré le succès de $\mathrm{M}$. Biya, son pouvoir se trouvait fragilisé avec seulement près de $40 \%$ des suffrages. A l'appel de ce dernier en vue d'un gouvernement non plus de coalition mais de large union, Bello aurait souhaité de véritables négociations où il devait avoir de portefeuilles ministériels au prorata de sa stature dans l'échiquier politique national et à l'hémicycle; donc deux ministres Kirdi respectivement du Nord et de l'Extrême-Nord, un du Littoral, un de l'Ouest, un du Centre-Sud et un du Sud-Ouest anglophone. Biya ne pouvait être qu'ulcéré de cette ambition nationale démesurée du Peul Bouba Bello Maïgari. En riposte, il négocia avec deux piliers de l'UNDP, MM. Hamadou Moustapha et Issah Tchiroma, deux Peul respectivement Premier Vice-Président et Secrétaire Général de l'UNDP qu'il nomma aux postes de Vice-Premier Ministre et Ministre.

Cette nomination vallut à Tchiroma et Hamadou Moustapha leur expulsion de l'UNDP par un communiqué en date du 23 juillet 1994, expulsion entérinée par un vote du Comité Central de l'UNDP en date des 21 et 22 janvier 1995. Depuis cette date ces deux ministres qui n'ont pas démissionné, ni avoir été démis, ne cessent de déstabiliser l'UNDP (tentative inf ructueuse de créer une «UNDP-Authentique», installation vaine des comités de base et de sections du partie, meetings etc...). Après avoir lutté sans succès contre l'UNDP de l'extérieur, ils se sont amendés pour saisir la justice afin que soient prononcées la nullité de la réunion du Comité Central de l'UNDP des 21 et 22 janvier ainsi que les résolutions qui ont sanctionné les travaux de cette instance du parti. Au mois d'Août 1995, ils ont rompu définitivement avec l'UNDP pour créer l'Alliance Nationale pour la Démocratie et le Progrès (ANDP). Seules les prochaines élections nous éclaireront sur leur poids politique réel.

L'impression qui se dégage de cet imbroglio politique est que dans une stratégie d'alliance avec les non-musulmans du Nord-Cameroun, Biya a voulu en nommant deux Peul de l'UNDP montrer aux yeux des Kirdi que ce parti n'était qu'une renaissance de l'hégémonie musulmane qu'il faut saborder à tout prix. A l'autre bout, la logique d'accumulation à animé Tchiroma et Hamadou Moustapha dès lors qu'ils n'étaient pas pressentis par Bello Bouba Maigari pour être proposés comme membres du gouvernement. Ils mènent une lutte sans

90

Source: Cameroon Tribune $n^{\circ} 5244$ du samedi 24 octobre 1992 
merci à Bello pour montrer à Biya qu'ils sont populaires, ce qui garantirait leur place au gouvernement.

\section{Réveil politique des populations Kirdi et la popularité grandissante du RDPC dans le Nord-Cameroun}

Ce parti recrute chez les populations Kirdi et les Arabes Choa. D'où son audience dans le Logone-et-Chari (Arabes Choa) où il avait raflé tous les quatre sièges aux législatives de 1992, dans les Monts Mandara densément peuplés (Mayo Sava, Mayo Tsanaga) avec sept élus sans oublier l'élu du Mayo Danaï. Lors du scrutin présidentiel, le score de M. Paul Biya était encore plus retentissant: $48 \%$ dans tout le Nord-Cameroun dont $26,13 \%$ dans la province de l'Adamaoua, 42,87\% dans le Nord et 47,65\% à l'Extrême-Nord.

Seulement, il est illusoire et excessif de dire avec Kees Schilder ${ }^{91}$ que ces résultats étaient surtout le reflet de la crainte d'une renaissance de l'hégémonie musulmane. Certes nous convenons avec lui que le soutien des chefs traditionnels et les manoeuvres de l'administration ont contribué à l'élection de M. Biya ainsi que l'a si bien démontré Achille Mbembe ${ }^{92}$. Mais le facteur ethnico-religieux n'est qu'un tremplin, un chenal par lequel est revendiquée la redistribution ainsi qu'un instrument d'accumulation.

Si nous prenons l'électorat Moundang du Mayo-Kani, contrairement à l'idée émise par Kees Schilder qui lie leur rapprochement à l'UNDP à leur ouverture à l'islam, une constante se dégage: en effet M. Helé Pierre, de l'ethnie Moundang, ancien ministre sous Ahidjo, membre du Comité Central de l'UNDP était le Directeur de campagne de M. Bouba Bello Maïgari à l'élection présidentielle. Et, par cette alliance les Moundang se voyaient déjà au pouvoir une fois ce dernier élu afin de contrôler les ressources rares de l'Etat camerounais. Ils avaient en souvenir le bon temps où Helé Pierre était aux commandes et ils souhaitaient son retour. Le même instinct d'accumulation a orienté le choix des Toupouri, Guiziga etc... dans leur alliance avec le RDPC.

Depuis son élection à la magistrature suprême en 1992, le Président Biya n'a effectué qu'une seule visite en province à l'Extrême-Nord les 19 et 20 avril 1995, la province qu'on qualifie de «fille aînée du Renouveau du Président Biya» comme on aime si bien le dire làbas. Cette visite a été celle du remerciement pour le soutien à l'élection présidentielle mais également celle du lancement de la campagne pour les futures échéances électorales, notamment les municipales qui tardent à venir depuis 1992.

91

Voir Kees Schilder, La démocratie aux champs..., op. cit. (note 83), p. 116.

92

Voir A. Mbembe, Crise de légitimité..., op. cit. (note 24), p. 349. 
Le MDR est né en 1991 à partir du Mouvement de Réflexion (groupe de fonctionnaires catholiques de Maroua, influencés idéologiquement par la DCK). Son Coordonnateur national, M. Dakolé Daïssala est un Toupouri originaire de l'Est du Département du Mayo Kani qui est avec le Mayo Danaï le fief du MDR. En vérité, il recrute principalement ses partisans chez les Toupouri, son audience auprès des Moundang, Massa et Moussey environnant étant limitée. Encore que les frictions entre Toupouri et Moundang sont légion et se reflètent dans des stéréotypes négatifs de part et d'autre et la désapprobation des mariages mixtes.

Pour ce, Dakolé apparaît moins qu'un leader Kirdi qu'un tribun toupouri, son ethnie dont il est le défenseur. C'est d'ailleurs même la substance de son discours dans son ouvrage précité:

«A présent, les partisans d'Ahidjo (Foulbé et autres assimilés) tiennent les Kirdi et plus spécialement les Toupouri pour responsables de l'échec du coup d'Etat et ne manquent pas de les accabler de leurs sarcasmes, assorties de menaces, de représailles, le cas échéant.

Certes, pareil comportement n'a aucune prise comme toute autre action destinée à les isoler du milieu des autres Nordistes, l'importance numérique et la conscience collective très robuste du groupe incriminé constituant une assurance et un rempart qui vient renforcer le puissant sentiment de justesse attaché à la cause défendue.

La vérité, c'est que l'importance démographique générale des Toupouri et leur goût naturel pour le métier des armes les ont placés, par la force des choses, au coeur de ces affrontements fratricides d'avril 1984, mais qu'ils étaient bien loin d'en être les mandants.» ${ }^{94}$

Nous avons relevé plus haut le rôle joué par Dakolé Daïssala pendant les élections de 1992 et les gains qui en ont résulté pour lui-même, pour les Kirdi et surtout pour les Toupouri. Il reste à préciser qu'il lutte moins pour la conquête du pouvoir que pour son partage en of frant une opposition de principe à l'UNDP, «vestige de l'U.C. d'Ahidjo».

L'expression est de G. Lavau qui, parlant du Parti Communiste Français, relève cette «fonction tribunitienne» qui est celle de défense et de représentation des couches «plébéiennes» de la société française. A l'instar du tribun de la plèbe, le PCF jouerait ce rôle. Ce faisant il «contribue objectivement à intégrer au système des couches sociales qui en étaient traditionnellement exclues» et à «consolider le système en détournant l'élan révolutionnaire potentiel des éléments marginaux vers des revendications plus limitées compatibles avec l'équilibre général de la société». Voir $G$. Lavau, A la recherche d'un cadre théorique pour l'étude du Parti Communiste Français, in: Revue Française de Science Politique, 1968, p. 445.

Dakolé Daïsala, op. cit. (note 19), p. 136. 
Il convient d'ailleurs de signaler la lutte larvée qui oppose chez les Toupouri Dakolé à M. Ayang Luc, membre du Comité Central du RDPC et Président du Conseil Economique et Social; querelles hégémoniques en vue de susciter les suffrages desdites populations. Le second, s'inquiétant de la popularité grandissante du premier devenu pour la circonstance son rival, reproche à ce dernier d'avoir extorqué pendant les législatives de 1992 les suffrages toupouri pour coopter des ministres d'ailleurs (quoique Kirdi) et non toupouri, A ce reproche, Dakolé répond par l'ambition d'implanter son parti dans tout le territoire national et l'indifférence d'Ayang face aux problèmes toupouri. On comprend, chacun veut marchander sa popularité à $\mathrm{M}$. Biya afin de rester aux commandes.

Au bout du compte, notre analyse visait à attirer l'attention sur le faux monolithe politique ahidjoïste du Septentrion camerounais. Comme toute politique d'exclusion, cet artifice n'a pas survécu après le règne de son auteur. ${ }^{95}$ Ensuite, et c'est l'élément le plus important, les Kirdi constituent un groupe très hétéroclite: ici, la concorde, la solidarité, ne sont toujours pas de mise et, la vote étant essentiellement un phénomène de groupe, on ne saurait parler de leur homogénéité politique. Au contraire, la plupart d'entre eux sont acquis à la cause du RDPC du Président Biya à travers leurs notables locaux dans un réseau de prédation, d'accumulation et de redistribution. Ce qui fragilise et réduit l'équation de popularité des leaders de partis kirdi à l'instar du MDR de Dakolé Daïssala. Le même phénomène est observé chez les ethnies Bamiléké de l'Ouest du pays rangées derrière le SDF du leader anglophone Ni John Fru Ndi.

Néanmoins, une recomposition politique kirdi susceptible d'intégrer ou de concurrencer véritablement l'UNDP dans la région est possible si le RDPC arrivait à perdre le pouvoir au Cameroun.

Sur l'échec de la politique d'unité nationale d'Ahidjo, voir E. Kengne Pokam, La problématique de l'unité nationale au Cameroun, Paris, 1986. Seulement l'auteur s'est laissé enfermé dans un ton raciste et belliciste à un portrait quasi narcissique de l'ethnie Bamiléké. Il est regrettable que rien n'ait été dit s'agissant de l'exclusion des autres ethnies à l'instar des Kirdi au Nord-Cameroun. 


\title{
The New Ethiopian Constitution and the Protection of Human Rights
}

\author{
By Heinrich Scholler
}

The new Ethiopian constitution of 1994 is not a direct result of the fall of the Mengistu regime. Some elements of the present constitution, such as the federal structure, the right to self determination of the ethiopian nations and nationalities and the guarantee of human rights, have already been part of the Transitional Period Charter, an interim constitution accepted on the London Conference in 1991. The charter followed the constitution of 1931, which had been revised in 1955 by Haile Selassie, and the Mengistu-constitution of 1987. Characteristic elements of the new constitution are the fundamental principles - especially the rule of law, ethnicity and self-determination, and human dignity as a foundation for freedoms and human rights - and a bill of rights guaranteeing traditional civil rights. The article compares the catalogue of rights guaranteed in the 1994 constitution with the rights enshrined in the International Covenant on Civil and Political Rights of 1966. It can be seen that there are greater similarities between the Constitution and the Covenant as compared to the African Charter on Human and Peoples' Rights. Unique about the 1994 constitution is the special attention given to the protection of the language, culture and autonomy of minorities. The article provides both a broad overview over the provisions of the constitution and the most important aspects contained in it.

\section{Ethnicity and Power in Northern Cameroon}

\section{By Ibrahim Mouiche}

Social and religious cleavages in Northern Cameroon are major factors for ethnic conflicts in this region with regard to the accumulation of power. Traditionally, Cameroon - which comprises 234 ethnic groups - is divided into a "Moslem North" and a "Christian and Animist South". This idea was cultivated by the regime of President Ahidjo, a Peul himself, and is widespread in the minds of Cameronians. For Ahidjo, the North which he originated from constituted a political base held together by the "islam way of life". The article argues that this was and is illusionary, since Northern Cameroon is multi-ethnic and the nonMoslems, commonly called "Kirdis" (pagans), make up for over two thirds of the population despite their being subject to moslem hegemony represented by the ethnic group of the Peul.

When President Paul Biya, a man of the South, came to power in 1982, his position was more and more questioned by a pro-Ahidjo moslem domination which he consequently undertook to dismantle with the help of the emancipated Kirdi. And since the restauration 
of multipartyism in 1990, the mostly christianized non-Moslems support President Biyas RDPC party and oppose the UNCP of the Peul Bello Bouba Maigari. Furthermore, the Kirdi constitute a very heterogeneous group in itself divided by numerous conflicts.

\section{Human Rights in Sub-Saharan Africa: Towards Complementary Enforcement of Social Justice}

\section{By Sufian Hemed Bukurura}

The past and present decades are said to epitomize the era of good governance and transparency. It is in that context that constitutionalism, human rights and democracy are contemporary subjects of discussion all over the world. These concepts mean a lot to the organization of societies in general and legal systems in particular.

The current economic, political and social set-up of Sub-Saharan Africa does not seem to ensure that constitutionalism, human rights and democracy are readily available for the enjoyment of all people. The paper is a modest attempt in marking some signposts towards the exploration of alternative enforcement mechanisms, considering, inter alia, public interest litigation, social action litigation and participatory human rights enforcement.

\section{The Constitition of Paraguay of 1992 and its Predecessors}

\section{By Anja Schoeller-Schletter}

After decades of authoritarian rule, brought to an end in 1989 by the overthrow of General Alfredo Stroessner who dominated the country's politics since 1954, Paraguay is experiencing a process of transition from military dictatorship to democracy. A new constitution, passed in 1992, laid the legal foundation for a democratic government. As a reaction to the deficits of the preceding constitutions of 1967 and 1940, executive power was considerably restricted, the legislature was strengthened and the judiciary was subjected to fundamental reforms in order to guarantee its independence. Regardless of all changes in the normative system, tradition in terms of political practices and social structure turns out to be an impediment to the process of democratization.

The survey focuses on the solutions of the constitution of 1992 for the normative and political problems of the constitution of 1967: the extension of human rights protection; the abolition of the presidential authority to dissolve the Congress and the control of Congress 